\title{
MOSCAS-DAS-FRUTAS (DIPTERA: TEPHRITIDAE) E SEUS PARASITÓIDES EM PLANTAS HOSPEDEIRAS DE TRÊS MUNICÍPIOS DO NORTE DO ESTADO DE MINAS GERAIS
}

\author{
C.D. Alvarenga ${ }^{1}$, C.A.R. Matrangolo ${ }^{1}$, G.N. Lopes ${ }^{1}$, M.A. Silva ${ }^{1}$, \\ E.N. Lopes $^{1}$, D.A. Alves ${ }^{1}$, A.S. Nascimento ${ }^{2}$, R.A. Zucchi ${ }^{3}$
}

1Universidade Estadual de Montes Claros, Departamento de Ciências Agrárias, Rua Reinaldo Viana, 2630, CEP 39440-000, Janaúba, MG, Brasil. E-mail: clarice.corsato@unimontes.br

\section{RESUMO}

\begin{abstract}
Olevantamento das moscas-das-frutas foi por meio de coleta de frutos em áreas rurais e urbanas de Jaíba, Janaúba e Nova Porteirinha, durante 50 meses (maio/1999 a abril/2001 e junho/2002 a julho/2004). Foram amostradas 32 espécies de frutos em 17 famílias, porém foram obtidas moscasdas-frutas em apenas 18 hospedeiros. Foram coletados exemplares de Ceratitis capitata (Wied.) e de oito espécies de Anastrepha: A. fraterculus (Wied.), A. obliqua (Macquart), A. sororcula Zucchi, A. turpiniae Stone, A. zenildae Zucchi, A. pickeli Lima, A. montei Lima e Anastrepha n.sp.3. C. capitata ocorreu principalmente em hospedeiros introduzidos e foi predominante em áreas urbanas. As espécies de Anastrepha predominaram em áreas rurais. Umbu (Spondias tuberosa Arr. Câm.) e goiaba (Psidium guajava L.) foram os hospedeiros mais infestados pelos tefritídeos. Sete espécies de parasitóides foram coletadas:Doryctobracon areolatus (Szépligeti)(mais numeroso), D. fluminensis (Lima), D. brasiliensis (Szépligeti), Opius bellus Gahan.,Utetes anastrephae (Viereck), Diachasmimorpha longicaudata (Ashmead) e Aganaspis pelleranoi (Brèthes).
\end{abstract}

PALAVRAS-CHAVE: Anastrepha, Ceratitis capitata, braconídeos, figitídeo.

\section{ABSTRACT}

FRUIT FLIES(DIPTERA:TEPHRITIDAE) AND THEIR PARASITOIDSINHOST PLANTSFROM THREE COUNTIES OF THE NORTH OF MINAS GERAIS STATE, BRAZIL. This fruit-fly species survey was based on fruits sampled at several sites (urban and rural areas) in the counties of Jaíba, Janaíba and Nova Porteirinha, in Minas Gerais State, Brazil over a 50-month period (from May 1999 to April 2001 and from June 2002 to July 2004). Thirty-two fruit species in 17 families were sampled, but fruit flies emerged from only 18 host-fruit species. Ceratitis capitata(Wied.) and eight Anastrepha species - A. fraterculus (Wiedemann), A. obliqua (Macquart), A. sororcula Zucchi, A. turpiniaeStone, A. zenildae Zucchi, A. pickeli Lima, A. montei Lima and Anastrepha n.sp.3 - were collected. Medflies emerged mostly from introduced fruits and were predominant in the urban areas, while the Anastrepha species were predominant in the rural areas. Spondias tuberosa Arr. Câm. ("umbu") and Psidium guajava L. (guava) were the hosts most infested by tephritid larvae. Seven fruit-fly parasitoid species were collected: Doryctobracon areolatus (Szépligeti) (more abundant), D. fluminensis (Lima), D. brasiliensis (Szépligeti), Opius bellus Gahan, Utetes anastrephae (Viereck), Diachasmimorpha longicaudata (Ashmead) and Aganaspis pelleranoi (Brèthes).

KEY WORDS. Anastrepha, Ceratitis capitata, braconids, figitid.

\section{INTRODUÇÃO}

O norte do Estado de Minas Gerais é uma região que tem se destacado consideravelmente pelo grande crescimento na exploração da fruticultura. O crescimento da agricultura irrigada no norte de Minas Gerais, a partir do início da década de 90, facilitado pelas condições edafoclimáticas, disponibilidade de crédito e oferta de tecnologias geradas e/ou adaptadas à região, permitiu a expansão da área cultivada com várias espécies de fruteiras e olerícolas. Atualmente, são cultivados na região cerca de 25.000 ha em áreas irrigadas, sendo 20.000 ha com fruticultura e 5.000 ha com olericultura (CODEVASF, 2006).

${ }^{2}$ Embrapa Mandioca e Fruticultura, Cruz das Almas, BA, Brasil.

${ }^{3}$ Universidade de São Paulo, Escola Superior de Agricultura “Luiz de Queiroz", Piracicaba, SP, Brasil. 
A incidência de moscas-das-frutas é problema sério nos perímetros irrigados do norte de Minas Gerais (Jaíba e Gorutuba), acarretando aumento nos custos de produção em razão das frequentes aplicações de inseticidas e perdas na produção. Com a ampliação das áreas cultivadas, há o aumento da população dessas pragas devido ao processo natural de dispersão e também pelo transporte involuntário de frutos infestados de uma região para outra (MorgAnTE, 1991), inclusive de áreas urbanas próximas aos pomares comerciais de áreas rurais.

Para se estabelecer qualquer programa de controle desses dípteros, torna-se necessário o prévio conhecimento das espécies, seus parasitóides e respectivas plantas hospedeiras. Portanto, o objetivo deste trabalho foi identificar as moscas-das-frutas e seus parasitóides em potenciais hospedeiros em três municípios da região norte de Minas Gerais.

\section{MATERIAL E MÉTODOS}

Os levantamentos foram conduzidos em pomares de três municípios do norte do Estado de Minas Gerais: Janaúba (área urbana), Nova Porteirinha (área rural do Projeto de Irrigação do Gorutuba) e Jaíba (área rural do Projeto de Irrigação do Jaíba, em Mocambinho), localizados na região semi-árida do Estado, com ecossistema conhecido como caatinga. Os locais de coleta foram: Jaíba - dois pomares comerciais de goiaba de 0,5 ha cada um, com áreas de mata e pomares domésticos adjacentes; pomar comercial de manga de 1 ha, com pomar doméstico adjacente; pomares comerciais de uva, pitanga, acerola, pinha e goiaba de 0,3 ha cada um e pomar comercial de acerola de 1 ha; Nova Porteirinha - três pomares comerciais de goiaba de 0,5 ha cada um, com áreas de mata e pomares domésticos adjacentes e três pomares comerciais de manga de 1,0 ha cada um, com pomares domésticos adjacentes; Janaúba - quatro pomares urbanos com diversas frutíferas.

Os levantamentos das moscas-das-frutas e parasitóides foram por meio da coleta de frutos maduros ou em amadurecimento, durante 50 meses (maio/ 1999 a abril/2001 e junho/2002 a julho/2004). Os frutos nas árvores foram coletados aleatoriamente em diferentes alturas da copa. Os frutos recém-caídos, que estavam em boas condições de conservação e sem orifícios de saída das larvas, também foram incluídos na coleta. O tamanho das amostras foi variável e dependente da disponibilidade de frutos

As amostras, devidamente rotuladas (data, local e coletor), foram acondicionadas em bandejas plásticas e transportadas ao Laboratório de Entomologia da Universidade Estadual de Montes Claros. Os frutos foram quantificados e acondicionados em recipientes plásticos contendo vermiculita umedecida e fechados com tecido voil e elástico. As amostras foram mantidas sob condições controladas de temperatura $\left(27 \pm 1^{\circ} \mathrm{C}\right)$.

Após sete a quinze dias, as larvas e/ou pupários foram retirados do fruto e da vermiculita e transferidos para frascos de vidro contendo vermiculita, para emergência dos adultos. Os insetos emergidos foram fixados emálcool $70 \%$ para posterior identificação. A associação da espécie de parasitóide e da mosca-dasfrutas somente foi considerada quando no frasco de criação havia emergência de uma única espécie de mosca e do parasitóide (LEONEL JUNIOR et al., 1996).

Oíndice de infestação por tefritídeos foi calculado dividindo-se o número de pupários obtidos pelo número de frutos coletados, de cada espécie de planta hospedeira. O parasitismo foi calculado pela fórmula: $\mathrm{P}=$ (no-de parasitóides $/$ total deadultos $) \times 100$, para cada espécie de hospedeiro.

As identificações das espécies de plantas foram realizadas com base na literatura e no material contido no Herbário do Laboratório deSementes e Botânica da UNIMONTES, Campus Janaúba.

Os insetos-testemunha estão depositados na coleção do Departamento de Ciências Agrárias da UNIMONTES.

\section{RESULTADOS}

\section{Moscas-das-frutas}

Nas áreas rural e urbana, foram coletados 60.281 frutos (1.838 amostras), pertencentes a 17 famílias e 32 espécies de plantas (22 introduzidas e 10 nativas). Foram obtidos 16.398 pupários em $18(52,78 \%)$ hospedeiros (12 introduzidos e seis nativos), dos quais emergiram 10.847 espécimes (moscas-das-frutas e parasitóides) (Tabela 1), com viabilidade pupal de $66,2 \%$. Das moscas emergidas, 8.941 pertenciam a Anastrepha (4.646 machos e 4.295 fêmeas) e 1.153 a Ceratitis capitata ( 565 machos e 588 fêmeas) (Tabela 2). Em pomares da área urbana, foram coletados 3.559 frutos (162 amostras) e foram obtidos 887 pupários, dos quais emergiram 57 espécimes de Anastrepha, 523 de C. capitata e 12 deDoryctobracon areolatus (Tabela 6). $\mathrm{Na}$ área rural, foram coletados 56.722 frutos (1.676 amostras) e foram obtidos 15.511 pupários, dos quais emergiram 8.884 espécimes de Anastrepha, 630 de $C$. capitata e 741 parasitóides. Em áreas urbanas, 90,2\% das moscas coletadas pertenciam a C. capitata e, em áreas rurais, 93,4\% ao gênero Anastrepha.

As maiores quantidades de pupários e emergência demoscas foramobtidas degoiaba (Psidiumguajava), umbu (Spondiastuberosa), mandioca (Manihotesculenta), pitanga (Eugenia uniflora), tangerina (Citrus nobilis), seriguela (Spondias purpurea), castanhola (Terminalia catappa) e juá (Ziziphus joazeiro) (Tabela 1). 


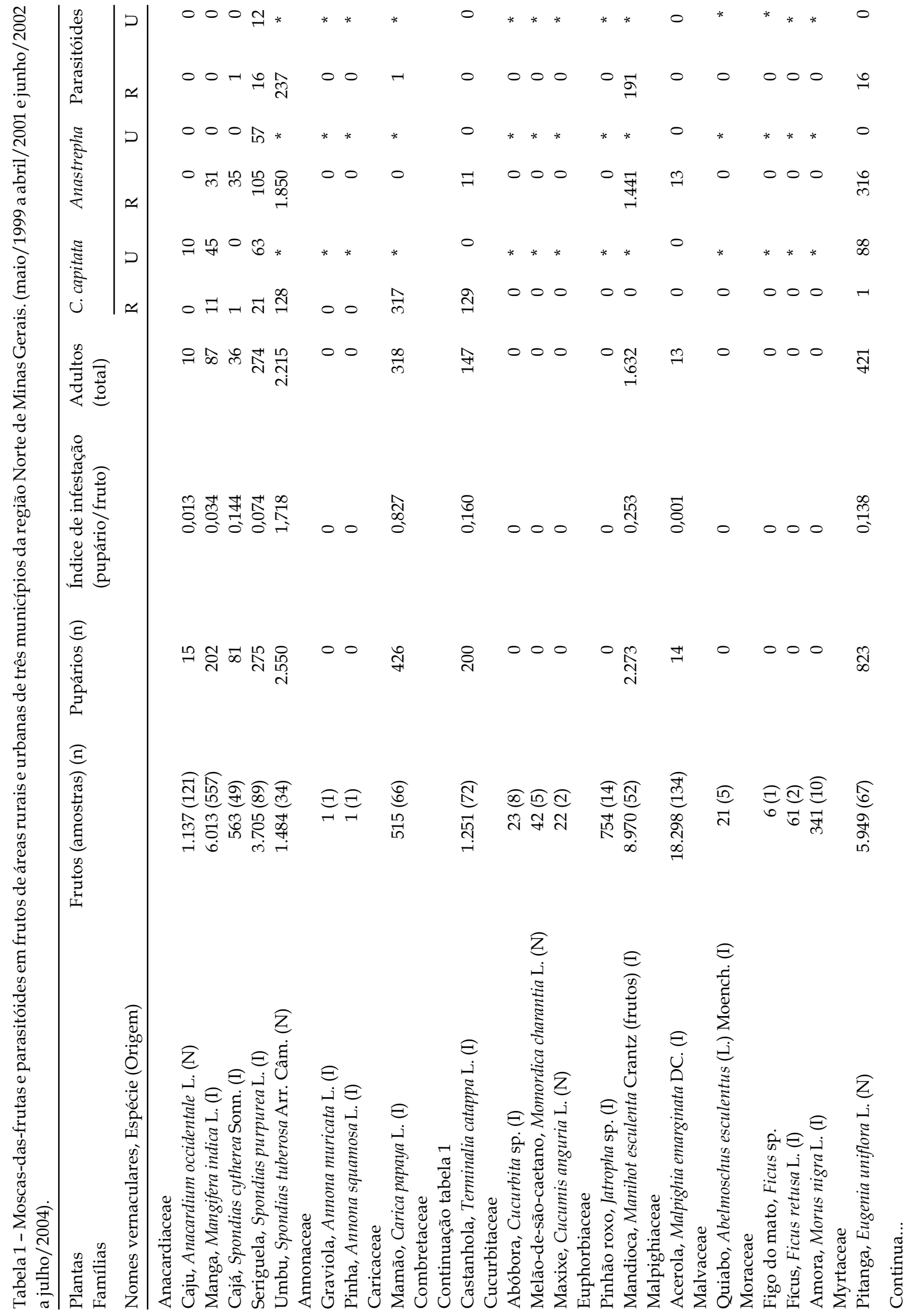




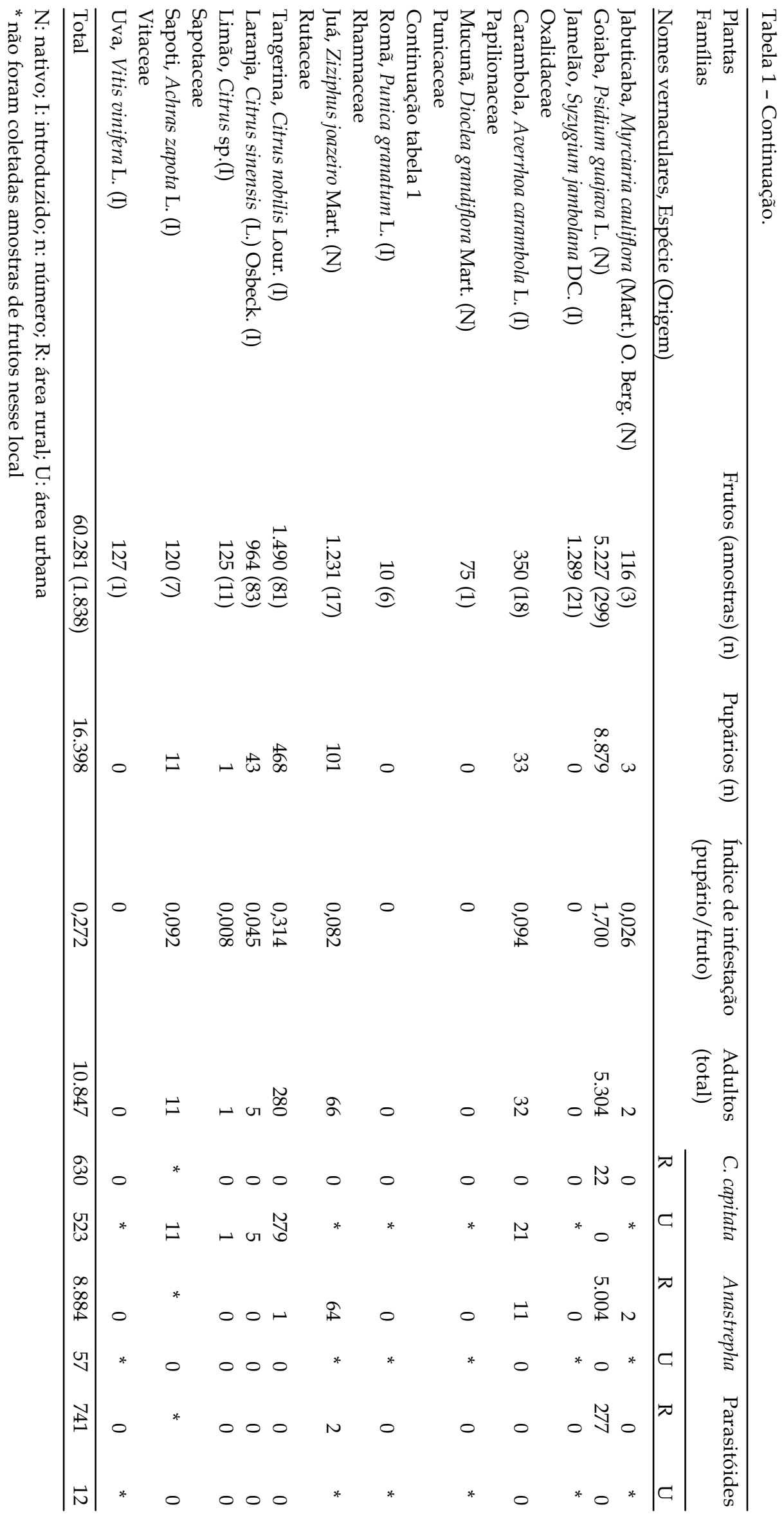


As espécies de Anastrephaforam observadas em 13 hospedeiros (oito introduzidos e cinco nativos). A. obliqua infestou o maior número de hospedeiros, sete das 13 espécies com larvas de moscas-das-frutas (Tabela 4). Todos os espécimes deAnastrephaemáreas urbanas foram obtidos exclusivamente de seriguela.

C. capitata foi associada a 14 espécies frutíferas $(10$ introduzidas e quatro nativas). Na área urbana, $C$. capitata infestou frutos de nove espécies (sete introduzidos e dois nativos) e na área rural, frutos de cinco espécies (três introduzidos e dois nativos).

\section{Parasitóides}

Das 1.838 amostras, em apenas 50 foram coletados parasitóides. Dos 16.398 pupários, emergiram 753 parasitóides: D. areolatus (588), D. fluminensis (97), D. brasiliensis (44), Diachasmimorpha longicaudata (20), Opius bellus (1), Utetes anastrephae (2) e Aganaspis pelleranoi (1) (Tabela 5).

Das oito espécies de frutíferas onde ocorreu parasitismo, três são Anacardiaceae [seriguela $(10,22 \%)$, umbú $(10,70 \%)$ e cajá $(2,77 \%)]$, duas Myrtaceae [goiaba $(4,39 \%)$ e pitanga (3,80\%)], uma Caricaceae (mamão:0,31\%), uma Euphorbiaceae (mandioca: 11,70\%) e uma Rhamnaceae (juá:3,03\%). Aproximadamente $39 \%$ dos parasitóides foram associados às larvas frugívoras em mirtáceas e aproximadamente 35\% em anacardiáceas (Tabela 1).

\section{DISCUSSÃO}

\section{Moscas-das-frutas}

As espécies de Anastrepha infestaram 13 espécies de frutos hospedeiros, entretanto, ao contrário do observado em outras regiões (NELOso et al., 2000), inclusive no semi-árido de Petrolina (HaJI; MiRANDA, 2000), não foi nítida a preferência dos hospedeiros nativos em relação aos introduzidos. Entretanto, $C$. capitata mostrou evidente preferência pelos hospedeiros introduzidos em relação aos nativos, como tem sido observado em várias regiões brasileiras (MALAVASI et al., 1980).
A presença das moscas-das-frutas nas áreas rural e urbana ocorre em razão da concentração dos seus hospedeiros favoritos em uma ou outra área (Tabela 1). Na região de Juazeiro/Petrolina, por exemplo, C. capitata predominou em relação às espécies de Anastrepha na área urbana, em razão da ocorrência do hospedeiro chapéu-de-sol (Terminalia catappa) (Nascimento; CARvalho, 2000). No norte de Minas, embora esse hospedeiro tenha sido amostrado em áreas rurais e urbanas, somente nas áreas rurais foi infestado por tefritídeos. Apesar de ter sido obtido mais exemplares de C. capitata em áreas rurais, essa espécie mostrou preferência pelas áreas urbanas, em razão do maior concentração de hospedeiros introduzidos. Consequentemente, em áreas urbanas, atacou um maior número de espécies frutíferas que nas áreas rurais. Aproximadamente $50 \%$ dos exemplares em áreas rurais foram obtidos exclusivamente em mamão, que apresentavam sintomas da meleira. Os mamões verdes infectados pelo vírus da meleira são suscetíveis ao ataque de C. capitata (HABibe et al., 2004). Por outro lado, os exemplares de Anastrepha foram obtidos de um único hospedeiro em áreas urbanas (seriguela), mostrando nítida preferência pelas áreas rurais. Em seriguela e pitanga, onde foram coletados tanto C. capitata quanto Anastrepha, nota-se que C. capitata predominou nas amostras das áreas urbanas e Anastrepha nas amostras das áreas rurais (Tabela 1 ).

O número de amostras dos hospedeiros foi variável, em razão da disponibilidade de frutos no campo (Tabelas 1 e 3). Devido às condições climáticas extremas da região (alta temperatura e baixa precipitação pluvial), algumas espécies de frutíferas possuem somente um ou alguns períodos muito curtos de frutificação no ano.

Alguns frutos já referidos como hospedeiros de moscas-das-frutas (ZucCHI, 2008) foram pouco infestados, como a carambola (Averrhoacarambola), a laranja (Citrus sinensis), a manga (Mangifera indica) e o sapoti (Achras zapota). É preciso ressaltar que esses hospedeiros frutificaram em períodos coincidentes com os hospedeiros mais atacados (goiaba e umbú) e às vezes em pomares irrigados, em épocas que outras plantas hospedeiras não frutificam (Tabela 3).

Tabela 2 - Moscas-das-frutas e parasitóides em três municípios do norte de Minas Gerais (maio/1999 a abril/2001 e junho/2002 a julho/2004).

\begin{tabular}{|c|c|c|c|c|c|c|c|c|c|c|}
\hline \multirow[t]{2}{*}{ Insetos } & \multicolumn{3}{|c|}{ Jaíba } & \multicolumn{3}{|c|}{ Janaúba } & \multicolumn{3}{|c|}{ Nova porteirinha } & \multirow[t]{2}{*}{ Total } \\
\hline & Macho & Fêmea & Total & Macho & Fêmea & Total & Macho & Fêmea & Total & \\
\hline Anastrepha & 2.902 & 2.602 & 5.504 & 4 & 53 & 57 & 1.740 & 1.640 & 3.380 & 8.941 \\
\hline C. capitata & 186 & 227 & 413 & 233 & 290 & 523 & 146 & 71 & 217 & 1.153 \\
\hline Parasitóides & 223 & 245 & 468 & 4 & 8 & 12 & 114 & 159 & 273 & 753 \\
\hline
\end{tabular}




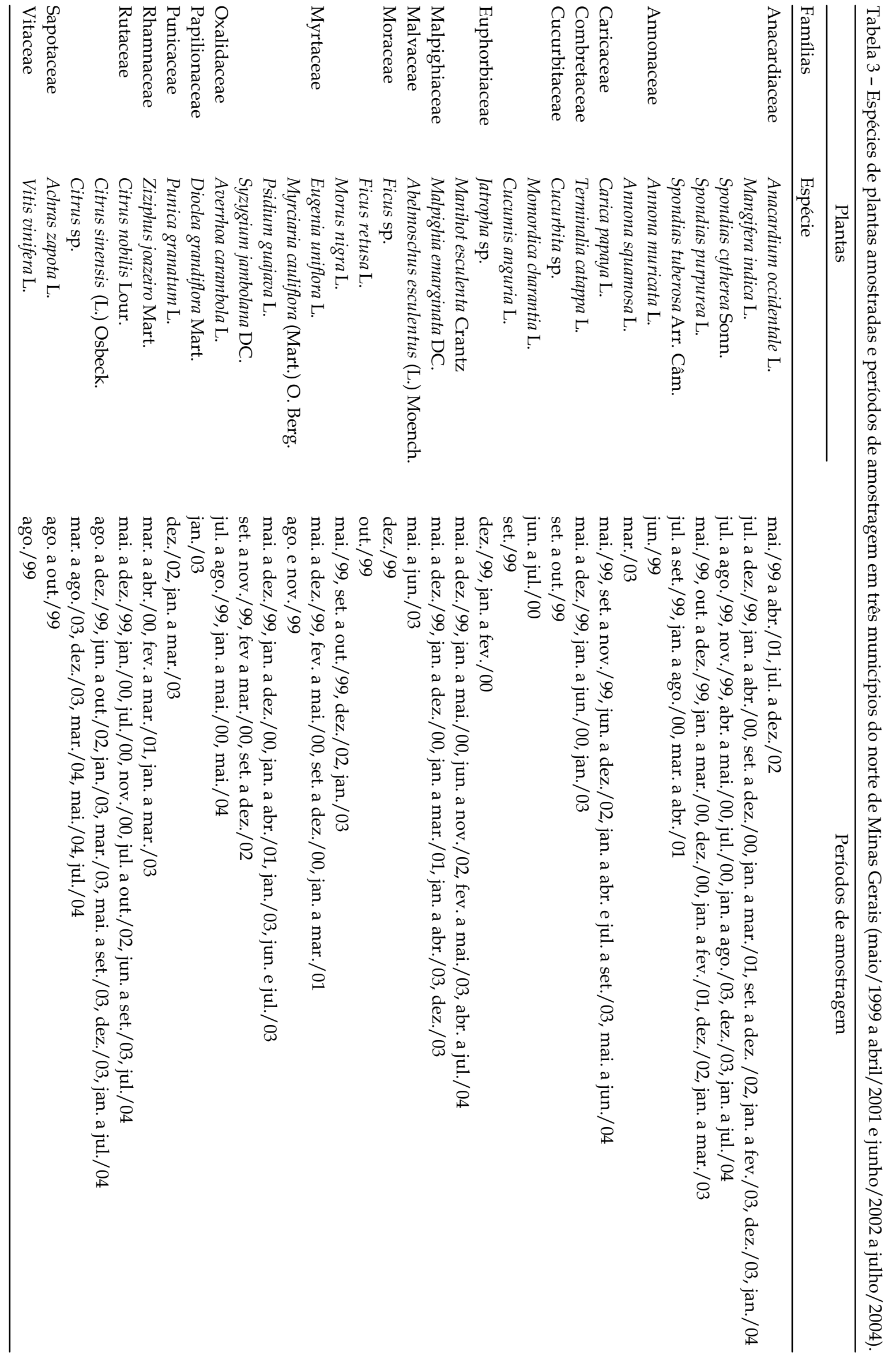


Tabela 4 - Espécies de Anastrepha e seus hospedeiros em Jaíba, Janaúba e Nova Porteirinha, MG (maio/1999 a abril/ 2001 e junho/2002 a julho/2004).

\begin{tabular}{|c|c|c|c|c|c|}
\hline \multicolumn{3}{|c|}{ Hospedeiros } & \multirow[t]{2}{*}{ Espécies de Anastrepha (fêmeas) } & \multirow[t]{2}{*}{ Machos } & \multirow[t]{2}{*}{ Total } \\
\hline Famílias & Espécies & $\begin{array}{l}\text { Nomes } \\
\text { vernaculares }\end{array}$ & & & \\
\hline \multirow[t]{10}{*}{ Anacardiaceae } & \multirow[t]{2}{*}{ Mangifera indica $\mathrm{L}$. } & \multirow[t]{2}{*}{ Manga } & Anastrepha fraterculus (Wied.) (6) & - & - \\
\hline & & & Anastrepha obliqua (Macquart) (10) & 15 & 31 \\
\hline & \multirow[t]{4}{*}{ Spondias purpurea $\mathrm{L}$. } & \multirow[t]{4}{*}{ Seriguela } & Anastrepha fraterculus (Wied.) (5) & - & - \\
\hline & & & Anastrepha obliqua (Macquart) (54) & - & - \\
\hline & & & Anastrepha sororcula Zucchi (3) & - & - \\
\hline & & & Anastrepha zenildae Zucchi (38) & 62 & 162 \\
\hline & \multirow[t]{3}{*}{ Spondias tuberosa Arr. Câm. } & \multirow[t]{3}{*}{ Umbu } & Anastrepha fraterculus (Wied.) (1) & - & - \\
\hline & & & Anastrepha obliqua (Macquart) (891) & - & - \\
\hline & & & Anastrepha zenildae Zucchi (7) & 651 & 1.850 \\
\hline & Spondias cytherea Sonn. & Cajá & Anastrepha obliqua (Macquart) (14) & 21 & 35 \\
\hline Combretaceae & Terminalia catappa L. & Castanhola & Anastrepha obliqua(Macquart) (1) & 10 & 11 \\
\hline Malpighiaceae & Malpighia emarginata DC. & Acerola & Anastrepha zenildae Zucchi (3) & 10 & 13 \\
\hline \multirow[t]{9}{*}{ Myrtaceae } & \multirow[t]{5}{*}{ Psidium guajava L. } & \multirow[t]{5}{*}{ Goiaba } & Anastrepha fraterculus (Wied.) (490) & - & -5.004 \\
\hline & & & Anastrepha obliqua (Macquart) (40) & - & - \\
\hline & & & Anastrepha sororcula Zucchi (97) & - & - \\
\hline & & & Anastrepha turpiniae Stone (6) & - & - \\
\hline & & & Anastrepha zenildae Zucchi (1.861) & 2.510 & 5.004 \\
\hline & Myrciaria cauliflora (Mart.) & Jabuticaba & Anastrepha sp. & 2 & 2 \\
\hline & O. Berg. & & & & \\
\hline & \multirow[t]{2}{*}{ Eugenia uniflora L. } & \multirow[t]{2}{*}{ Pitanga } & Anastrepha fraterculus (Wied.) (131) & - & - \\
\hline & & & Anastrepha sororcula Zucchi (2) & 183 & 316 \\
\hline Oxalidaceae & Averrhoa carambola L. & Carambola & Anastrepha obliqua (Macquart) (6) & 5 & 11 \\
\hline Rhamnaceae & Ziziphus joazeiro Mart. & Juá & Anastrepha zenildae Zucchi (27) & 37 & 64 \\
\hline
\end{tabular}

Tabela 5 - Espécies de parasitóides e seus hospedeiros em Jaíba, Janaúba e Nova Porteirinha, MG (maio/1999 a abril/ 2001 e junho/2002 a julho/2004).

\begin{tabular}{lrll}
\hline Espécies de parasitóides & No & $\begin{array}{l}\text { Plantas } \\
\text { associadas }\end{array}$ & Insetos hospedeiros \\
\hline Doryctobracon areolatus (Szépligeti) & 277 & Goiaba & C. capitata, A. zenildae, A. fraterculus, A. sororcula \\
& 2 & Juá & A. zenildae \\
& 28 & Seriguela & A. obliqua, A. zenildae \\
& 1 & Mamão & C. capitata \\
& 45 & Mandioca & A. pickeli \\
& 234 & Umbu & C. capitata, A. obliqua \\
& 1 & Cajá & A. obliqua \\
Doryctobracon fluminensis (Lima) & 97 & Mandioca & A. pickeli, A. montei, Anastrepha n.sp.3 \\
Doryctobracon brasiliensis (Szépligeti) & 44 & Mandioca & A. pickeli, A. montei, Anastrepha n.sp.3 \\
Diachasmimorpha longicaudata (Ashmead) & 3 & Mandioca & A. pickeli, A. montei \\
& 16 & Pitanga & A. fraterculus \\
Opius bellus Gahan & 1 & Umbu & A. obliqua \\
Utetes anastrephae (Viereck) & 1 & Mandioca & A. pickeli \\
& 1 & Mandioca & A. pickeli \\
Aganaspis pelleranoi (Brèthes) & 1 & Umbu & A. obliqua \\
Total & 1 & Umbu & A. obliqua \\
\hline
\end{tabular}


Tabela 6 - Moscas-das-frutas e parasitóides coletados em áreas rurais e urbanas no norte de Minas Gerais (maio/1999 a abril/2001 e junho/2002 a julho/2004).

\begin{tabular}{|c|c|c|c|c|c|c|c|}
\hline \multirow[t]{2}{*}{ Insetos } & \multicolumn{3}{|c|}{ Área rural } & \multicolumn{3}{|c|}{ Área urbana } & \multirow[t]{2}{*}{ Total } \\
\hline & Macho & Fêmea & Total & Macho & Fêmea & Total & \\
\hline Anastrepha & 4.642 & 4.242 & 8.884 & 4 & 53 & 57 & 8.941 \\
\hline C. capitata & 299 & 331 & 630 & 233 & 290 & 523 & 1.153 \\
\hline Parasitóide & 337 & 404 & 741 & 4 & 8 & 12 & 753 \\
\hline
\end{tabular}

Como observado por vários autores, foi confirmada a preferência de A. obliqua por anacardiáceas. Entretanto, diferentemente do constatado em levantamentos na região (CANAl et al., 1998a,b; AlvarengA, 2004), foi observada infestação significativa em goiaba (Psidium guajava) por A. obliqua (Tabela4). Agoiaba é amplamente cultivada nos perímetros irrigados e frutifica durante todo o ano, sendo infestada principalmente por $A$. zenildaee $A$. fraterculus e, em segundo plano, por A. sororcula, A. obliqua, A. turpiniae e $C$. capitata (Tabela 4), como observado também por Alvarenga (2004). A. zenildae foi a única espécie associada ao juá (Ziziphus joazeiro), o que pode indicar uma especificidade desse tefritídeo com hospedeiros da família Rhamnaceae (URAмото et al., 2008).

Além da sucessão hospedeira, outro fator que concorre para o aumento da densidade populacional de moscas-das-frutas é a existência de diversos ciclos de frutificação de um mesmo hospedeiro. No semiárido do norte mineiro, esse fato favorece a população das moscas-das-frutas, pois há diversas espécies de frutíferas irrigadas em áreas urbanas, que frutificam durante todo o ano, por exemplo, goiaba e pitanga (Tabela 3). Nessas áreas, por exemplo,A. obliquainfesta preferencialmente o umbu-cajá, que frutifica no mínimo seis meses por ano e, posteriormente, migra para a seriguela. Esses hospedeiros mantêm elevada a população de $A$. obliqua, que poderá atacar a goiaba e a manga, as quais são cultivadas comercialmente nos perímetros irrigados no norte de Minas Gerais.

\section{Parasitóides}

A quantidade de parasitóides obtidos foi diretamente dependente do nível de infestação do hospedeiro pelas larvas, ou seja, nos hospedeiros com mais larvas de moscas (goiaba e umbu), emergiram mais parasitóides (Tabela1).Osaltosíndices de parasitismo nas larvas em seriguela $(10,22 \%)$, umbu $(10,7 \%)$ e mandioca $(11,70 \%)$ resultaram das características desses frutos - epicarpo e mesocarpo finos - que favorecem o parasitismo. A espessura da polpa dos frutos atua como barreira ao parasitismo de larvas de moscas-das-frutas (HiCKEL, 2002).
Entre as espécies nativas, na área rural,D.areolatus infestou mais espécies de moscas-das-frutas em vários hospedeiros (Tabela 5), como tem sido observado em diversas localidades brasileiras (CANAL; ZUCCHI, 2000). A supremacia desse parasitóide tem sido observada ao longo de sua distribuição geográfica, provavelmente por possuir ovipositor longo, que possibilita encontrar as larvas nos mais diversos. $\mathrm{O}$ comprimento do ovipositor na eficiência do parasitismo pode ser reforçado pelos dados obtidos com $D$. longicaudata. Esse parasitóide exótico (liberações esporádicas na região) parasitóide parasitou larvas em mais plantas hospedeiras do que as espécies nativas, que possuem ovipositores curtos. Os parasitóides com ovipositores longos parasitam larvas em frutos pequenos e grandes, mas os parasitóides com ovipositores curtos limitam-se ao parasitismo de larvas em frutos pequenos (SIVINSKI et al., 1997).

Das 17 famílias de plantas amostradas, os parasitóides foram associados às espécies de apenas cinco famílias. A maior quantidade de parasitóides emergiu de goiaba (Myrtaceae) e de umbu (Anacardiaceae), cujas larvas foram parasitadas por D. areolatus. Em Santa Catarina, também foi constatada a atração dos parasitóides por mirtáceas e anacardiáceas (GARCIA, 2002). Entretanto, a maior diversidade de parasitóides foi obtida nos frutos de mandioca, que foi o único hospedeiro com emergência das seis espécies de braconídeos, sendo que duas - D. brasiliensis e D. fluminensis - foram coletados somente nesse hospedeiro (Tabela 5).

O parasitismo em pomares da área urbana foi bem reduzido quando comparado com a área rural (Tabela 6). Foram obtidos apenas 12 espécimens de D. areolatus associados às larvas em seriguela, único hospedeiro infestado por Anastrepha na área urbana.

O parasitismo total foi reduzido $(6,9 \%)$, sendo maior na área rural $(7,79 \%)$ e bem reduzido na área urbana (2,07\%) (Tabelas 1 e 6). Como observado por outros autores o parasitismo natural em regiões de semi-árido é baixo, em razão provavelmente das condições adversas do clima semi-árido (ARAújo;ZuCCHI, 2002; Cova; Bittencourt, 2003; Carvalho, 2004). 


\section{CONCLUSÕES}

Anastrepha frateculus, A. obliqua, A. sororcula, A. turpiniae, A zenildae, A. pickeli, A. montei, Anastrepha n.sp.3 e $C$. capitata são as espécies de moscas-dasfrutas associadas às plantas hospedeiras no norte de Minas Gerais.

Umbu (Spondias tuberosa) e goiaba (Psidiumguajava) são os hospedeiros mais infestados por moscas-dasfrutas no norte de Minas Gerais.

C. capitata é predominante na área urbana, associada principalmente a hospedeiros introduzidos; as espécies de Anastrepha predominam nos pomares de áreas rurais.

Os braconídeos Diachasmimorpha longicaudata, Doryctobracon areolatus, D. fluminensis, D. brasiliensis, Opius bellus e Utetes anastrephae o figitídeo Aganaspis pelleranoi parasitam moscas-das-frutas no norte de Minas Gerais.

O parasitismo natural de moscas-das-frutas no norte de Minas Gerais é baixo.

\section{AGRADECIMENTOS}

A Fundação de Amparo à Pesquisa de Minas Gerais (FAPEMIG) pelo apoio financeiro à pesquisa e pelas bolsas de estudos concedidas aos autores (Doutorado, Iniciação científica e BIPDT).

\section{REFERÊNCIAS}

ALVARENGA, C.D. Moscas-das-frutas (Diptera: Tephritidae) em pomares de goiaba no norte de Minas Gerais: Biodiversidade, parasitóides e controle biológico. 2004. 83f. Tese (Doutorado em Entomologia) - Escola Superior de Agricultura "Luiz de Queiroz", Universidade de São Paulo, Piracicaba, 2004.

ARAUJO, E.L.; ZUCCHI, R.A. Parasitóides (Hymenoptera: Braconidae) de moscas-das-frutas (Diptera: Tephritidae) na região de Mossoró/Assú, Estado do Rio Grande do Norte. Arquivos do Instituto Biológico, São Paulo, v.69, n.2, p.65-68, 2002.

CANAL, N.A.; ALVARENGA, C.D.; ZUCCHI, R.A. Análise faunística das espécies de moscas-das-frutas (Dip., Tephritidae) em quatro municípios do Norte do Estado de Minas Gerais. Scientia Agricola, v.55, n.1, p.1524, 1998a.

CANAL, N.A.; ALVARENGA, C.D.; ZUCCHI, R.A. Níveis de infestação de goiaba por Anastrepha zenildae Zucchi, 1979 (Dip., Tephritidae), em pomares comerciais do Norte de Minas Gerais. Anais da Sociedade Entomológica do Brasil, v.27, n.4, p.657-661, 1998b.
CANAL, N.A.; ZUCCHI R.A. Parasitóides - Braconidae. In: MALAVASI, A.; ZUCCHI R.A. (Ed.), Moscas-das-frutas de importância econômica no Brasil: conhecimento básico e aplicado. Ribeirão Preto: Holos, 2000. cap.15, p.119-126.

CARVALHO, R.S. Monitoramento de parasitóides nativos e de tefritídeos antes da liberação de Diachasmimorpha longicaudata (Hymenoptera: Braconidae) no Submédio São Francisco. Cruz das Almas: Embrapa, 2004. 6p. (Comunicado Técnico 100).

COMPANHIA DE DESENVOLVIMENTO DOS VALES DE SÃO FRANCISCO DO PARNAÍBA. (Brasil). Norte de Minas. 2006. Disponível em: <http:/ / www.codevasf.g ov.br/principal/perimetros-irrigados/polos-de-desenvolvimento-1/norte-de-minas>. Acesso em: 13 mai. 2009.

COVA, A.K.W.; BITTENCOURT, M.A.L. Ocorrência de moscas-das-frutas (Tephritidae) e parasitóides em frutos da região do Semi-Árido da Bahia. Magistra, v.15, p. 1-4, 2003. Especial entomologia.

GARCIA, F.R.M. Dinâmica populacional de moscas-dasfrutas (Diptera, Tephritidae) e ocorrência de seus parasitóides (Hymenoptera) na região Oeste de Santa Catarina. 2002. 99f. Tese (Doutorado em Biociências - Área de Zoologia) Pontifica Universidade Católica do Rio Grande do Sul, Porto Alegre, 2002.

HABIBE, T.C.; NASCIMENTO, A.S.; MALAVASI, A.; BRITO, D.B.; NETO, C.S. Comprovação da suscetibilidade de frutos de mamoeiros infectados pela meleira, à mosca-das-frutas (Ceratitis capitata). Cruz das Almas: Embrapa, 2004. 4p. (Comunicado Técnico 99).

HAJI, F.N.P.; MIRANDA, I.G. Pernambuco. In: MALAVASI, A.; ZUCCHI, R.A. (Ed.). Moscas-das-frutas de importância econômica no Brasil: conhecimento básico e aplicado. Ribeirão Preto: Holos, 2000. cap.33, p.229-233.

HICKEL, E.R. Espessura da polpa como condicionante do parasitismo de mosca-das-frutas (Diptera: Tephritidae) por Hymenoptera:Braconidae. Ciência Rural, v.32, n.6, p.1005-1009, 2002.

LEONEL JUNIOR., F.L.; ZUCCHI, R.A.; CANAL D.N. Parasitismo de moscas-das-frutas (Diptera. Tephritidae) por Braconidae (Hymenoptera) em duas localidades do estado de São Paulo. Anais da Sociedade Entomológica do Brasil, v.25, p.199-206, 1996.

MALAVASI, A.; MORGANTE, J.S.; ZUCCHI, R.A. Biologia de moscas-das-frutas (Diptera: Tephritidae). I. Lista de hospedeiros e ocorrência. Revista Brasileira de Biologia, v.40, p.9-16, 1980.

MORGANTE, J.S. Moscas-das-frutas (Tephritidae): características biológicas, descrição e controle. Brasília: SENIR, 1991. 19p. (Boletim Técnico de Recomendações para os Perímetros Irrigados do Vale São Francisco, 2). 
NASCIMENTO, A.S.; CARVALHO, R.S. Bahia. In: MALAVASI, A.; ZUCCHI, R.A. (Ed.). Moscas-das-frutas de importância econômica no Brasil: conhecimento básico e aplicado. Ribeirão Preto: Holos, 2000. cap.34, p.235-239.

SIVINSKI, J.; ALUJA M.; LOPÉZ M. Spatial and temporal distribution of parasitoids of Mexican Anastrepha species (Diptera: Tephritidae) within the canopies of fruit trees. Annals of the Entomological Society of America, v.90, p.604-618, 1997.

URAMOTO, K; MARTINS, D.S.; ZUCCHI, R.A. Fruit flies (Diptera, Tephritidae) and their associations with native host plants in a remnant area of the highly endangered Atlantic Rain Forest in the State of Espírito Santo, Brazil. Bulletin of Entomological Research, v.98, p.457-466, 2008.
VELOSO, V.R.S.; FERNANDES, P.M.; ZUCCHI, R.A. Goiás. In: MALAVASI, A.; ZUCCHI, R.A. (Ed.). Moscasdas-frutas de importância econômica no Brasil: conhecimento básico e aplicado. Ribeirão Preto: Holos, 2000. cap.36, p.247-252.

ZUCCHI, R.A. Fruit flies in Brazil - Anastrepha species and their hosts plants. 2008. Disponível em: http:/ / www. lef.esalq.usp.br/anastrepha>. Acesso em: 17 mai 2009).

Recebido em 18/2/08

Aceito em 8/6/09 